${ }^{\circ}$ Entomologica Fennica. 13 November 2000

\title{
New Records of Lepidoptera from the Kola Peninsula, Northwestern Russia
}

\author{
Mikhail V. Kozlov, Jukka Jalava \& Elena Shutova
}

Kozlov, M. V., Jalava, J. \& Shutova, E. 2000: New Records of Lepidoptera from the Kola Peninsula, Northwestern Russia. - Entomol. Fennica 11: 131-136.

We report 91 species of Lepidoptera discovered from the Kola Peninsula for the first time and confirm records of 15 species from which our earlier information was based solely on the literature. Tinea svenssoni Opheim, 1965, Biselachista kebneella Traugott-Olsen \& Nielsen, 1977 and Apotomis fraterculana Krogerus, 1945 are reported for the first time from Russia. The Lepidopteran fauna of the Kola Peninsula now numbers 676 species.

Mikhail V. Kozlov, Section of Ecology, FIN-20014 University of Turku, Finland Jukka Jalava, Zoological Museum, P.O. Box 17, FIN-00014 University of Helsinki, Finland

Elena V. Shutova, State Nature Reserve, Lineynaya str. 35, Kandalaksha, Murmansk reg., 184040 Russia

Received 30 June 1999, accepted 4 November 1999

\section{Introduction}

585 species of Lepidoptera were reported from the Kola peninsula in Kozlov \& Jalava (1994). Since then, several faunistic collecting trips have been made to poorly known areas and ecological samples have been collected yearly within the polluted areas near Monchegorsk. New data is included from the formerly unknown collection of the Kandalaksha Nature Reserve (SW-corner of the peninsula). Alltogether, about 20000 specimens were identified. About 8000 of these are preserved in collections of the Zoological Museum of Helsinki and the Kandalaksha State Reserve. In this paper we report 91 species for the first time from the Kola Peninsula and give a list of fifteen species of which our earlier information was based on literature.
We continue using numeric codes of the localities (Table 1) as in our 1994 paper, adding the new localities to the end (nos 80-86). The archipelago in Kandalaksha Bay was given a double code, a number for the archipelago and an abbreviated name for the separate island (Table 2). The nomeclature and systematics are according to Varis et al. 1995.

\section{List of new species}

\section{Nepticulidae}

Stigmella myrtillella (Stt.) 61: 1 ex. 5.7.1997. Ectoedemia minimella (Zett.) 26: 1 ex. 9.7. 1995. 
Table 1. The collecting localities in UTM grid and in biogeographical provinces

\begin{tabular}{llll} 
No & Locality name & UTM square & Province \\
\hline 9 & Salmijärvi & VC2 & Lps \\
26 & Dalnije Zelentsy & DS1 & Lmur \\
38 & St.Petersburg road N of 391 & WA2 & Lim \\
39 & Montshegorsk & WA2 & Lim \\
43 & St.Petersburg road S of 391 & VA4 & Lim \\
$44 a$ & Tshuna-tundra mt. & VA4 & Lim \\
45 & Jokostrov (15 km E Apatity) & WV1 & Lim \\
55 & Apatity & WV1 & Lim \\
$56 a$ & 15 km E Kirovsk & WV1 & Lim \\
60 & Kandalaksha & VV4 & Lim \\
61 & Luvenga & VV4 & Lim \\
63 & Zelenoborskij & VV4 & Kk \\
66 & Kovda (=Kouta) & VU3 & Kk \\
68 & Turij Pns. (20 km E Umba) & WU3 & Lim \\
72 & Salnitsa (74 km SE Umba) & XU1 & LV \\
74 & Varzuga & CP1 & LV \\
80 & Korchozero & VU2 & Kk \\
81 & Oleniy Archipelague & VV4 & Lim \\
82 & Luvenga Archipelague & VV4 & Lim \\
83 & Northern Archipelague & VV4 & Lim \\
84 & Porja Guba & WV2 & Lim \\
85 & Velikij Island Region & WU1 & Lim \\
87 & 30 km E Kovdor & VV1 & Lim \\
88 & 63 km E Kovdor & VA2 & Lim \\
\hline
\end{tabular}

${ }^{1}$ Localities 38 and 43 represent two pollution gradients directed $\mathrm{N}$ and $\mathrm{S}$ (respectively) of the Monchegorsk smelter along the road St.Petersburg-Murmansk. The second figure (after hyphen) shows the distance (in kms) from the smelter.

Table 2. Basic characteristics of the collecting sites on islands of Kandalaksha reserve.

\begin{tabular}{|c|c|c|c|c|c|}
\hline No & Abbr. & Locality & Latitude (N) & Longitude (E) & Area (ha) \\
\hline 81 & OL & Oleniy Is. & $67^{\circ} 05^{\prime} 44^{\prime \prime}$ & $32^{\circ} 23^{\prime} 27^{\prime \prime}$ & 875.2 \\
\hline 82 & BV & Berezhnoy Vlasov Is. & $67^{\circ} 05^{\prime} 04^{\prime \prime}$ & $32^{\circ} 41^{\prime} 33^{\prime \prime}$ & 102.9 \\
\hline 82 & $\mathrm{KR}$ & Krasotka Is. & $67^{\circ} 0448$ & $32^{\circ} 4333$ & 0.8 \\
\hline 83 & A1 & Aphanaska I Luda & $67^{\circ} 03^{\prime} 14^{\prime \prime}$ & $32^{\circ} 33^{\prime} 56^{\prime \prime}$ & 0.6 \\
\hline 83 & $\mathrm{~A} 2$ & Aphanaska II Luda & $67^{\circ} 03^{\prime} 04^{\prime \prime}$ & $32^{\circ} 33^{\prime} 58^{\prime \prime}$ & 0.7 \\
\hline 83 & AN & Anisimov Is. & $67^{\circ} 04^{\prime} 20^{\prime \prime}$ & $32^{\circ} 32^{\prime} 35^{\prime \prime}$ & 67.1 \\
\hline 83 & $\mathrm{BL}$ & Lomnishnyi Bolshoj Is. & $66^{\circ} 58^{\prime} 14^{\prime \prime}$ & $32^{\circ} 37^{\prime} 35^{\prime \prime}$ & 44.6 \\
\hline 83 & BS & Sennuk Bol'sja Luda & $66^{\circ} 57^{\prime} 26^{\prime \prime}$ & $32^{\circ} 41^{\prime} 58^{\prime \prime}$ & 1.6 \\
\hline 83 & $\mathrm{GO}$ & Gorelyi Is. & $67^{\circ} 03^{\prime} 40^{\prime \prime}$ & $32^{\circ} 36^{\prime} 23^{\prime \prime}$ & 6.9 \\
\hline 83 & $\mathrm{DE}$ & Devichja Luda & $66^{\circ} 59^{\prime} 20^{\prime \prime}$ & $32^{\circ} 36^{\prime} 33^{\prime \prime}$ & 11.3 \\
\hline 83 & LO & Lodejnyi Is. & $67^{\circ} 02^{\prime} 38^{\prime \prime}$ & $32^{\circ} 35^{\prime} 00^{\prime \prime}$ & 97.4 \\
\hline 83 & ML & Lomnishnyi Malyi Is. & $66^{\circ} 58^{\prime} 16^{\prime \prime}$ & $32^{\circ} 36^{\prime} 27^{\prime \prime}$ & 22.0 \\
\hline 83 & MM & Medvezhyi Malyi Is. & $66^{\circ} 59^{\prime} 16^{\prime \prime}$ & $32^{\circ} 40^{\prime} 06^{\prime \prime}$ & 12.2 \\
\hline 83 & RJ & Rjashkov is. & $67^{\circ} 02^{\prime} 56^{\prime \prime}$ & $32^{\circ} 33^{\prime} 37^{\prime \prime}$ & 396.7 \\
\hline 83 & SK & Skalistaja Luda & $67^{\circ} 03^{\prime} 32^{\prime \prime}$ & $32^{\circ} 35^{\prime} 41^{\prime \prime}$ & 0.1 \\
\hline 83 & TS & Tsentralnaja Luda & $67^{\circ} 03^{\prime} 10^{\prime \prime}$ & $32^{\circ} 35^{\prime} 52^{\prime \prime}$ & 0.7 \\
\hline 83 & TY & Tsyp-Luda & $67^{\circ} 02^{\prime} 48^{\prime \prime}$ & $32^{\circ} 35^{\prime} 58^{\prime \prime}$ & 0.2 \\
\hline 83 & VR & Voroniy Is. & $67^{\circ} 01^{\prime} 28^{\prime \prime}$ & $32^{\circ} 37^{\prime} 25^{\prime \prime}$ & 96.6 \\
\hline 84 & GO & Gorelyi Is. & $66^{\circ} 25^{\prime} 48^{\prime \prime}$ & $33^{\circ} 46^{\prime} 27^{\prime \prime}$ & 70.7 \\
\hline 84 & ST & Stolbovaja 1 Luda & $66^{\circ} 40^{\prime} 54^{\prime \prime}$ & $33^{\circ} 47^{\prime} 00^{\prime \prime}$ & 1.3 \\
\hline 85 & VE & Velikyi Is. & $66^{\circ} 33^{\prime} 57^{\prime \prime}$ & $33^{\circ} 20^{\prime} 00^{\prime \prime}$ & 7340.0 \\
\hline
\end{tabular}




\section{Prodoxidae}

Lampronia fuscatella (Tgstr.) 39: 1 ex. 29.6. 1994.

\section{Psychidae}

Dahlica charlottae (Meier) 43-65: 2 exx. 1.7. 1998; 43-35: 1 ex. 17.6.1999.

Psyche casta (Pallas) 66: 1 ex. 27.7.1996.

Psyche norvegica (Schöyen) 44a: $700 \mathrm{~m} .1$ ex. 17.7.1994, flying early (05.00 a.m.) in the morning.

Sterrhopterix standfussi (Wck.) 44a: $700 \mathrm{~m}$. 1 ex. 17.7.1994.

\section{Tineidae}

Niditinea striolella (Matsum.) 38-11: 1 ex. 20. 7.1997.

Niditinea truncicolella (Tgstr.) 61: 1 ex. 19.7. 1995.

Tinea trinotella Thnbg. 83-RJ: 2 exx. 2.7.1991, 12.7.1997.

Tinea bothniella Svensson. 84-GO: 1 ex. 24.7. 1991. Described from Sweden in 1953. Zaguljaev (1960) described two taxons (T. unidentella and $T$. sibiriella), which were later synonymized with T. bothniella (eg. Petersen, 1973; Ponomarenko, 1998). These synonymizations enlarge the distribution area of bothniella eastwards to Baikal and Mongolia.

Tinea svenssoni Opheim. 83-LO: 1 ex. 1.8. 1998. This species was separated from Tinea columbariella by Opheim in 1965 from Norway and Sweden. After that it has been recognized widely from the Nordic and the Baltic countries (Karsholt \& Razowski 1996) and is most probably distributed further to the east in the taiga zone. The larva lives in birds' nests (Jalava 1980). New for Russia.

\section{Gracillariidae}

Caloptilia elongella (L.) 84-GO: 1 ex. ex larva from Alnus 5.9.1993.

Phyllonorycter corylifoliellus (Hb.) 43-15: 1 ex. 30.6.1998; 82-KR: 1 ex. 11.6.1995.

\section{Yponomeutidae}

Swammerdamia compunctella(H.-S.) 61: 1 ex. 19.7.1995.

Cedestis farinatella (Dup.) 61: 4 exx. 27.8, 7.9.1997, 6.8.1998.

\section{Argyresthiidae}

Argyresthia goedartella (L.) 39-1: 1 ex. 28.6.1999.

\section{Plutellidae}

Ypsolopha parenthesella (L.) 61: light-trap, 22 exx. 23.8-10.9.1994-98.

Ypsolopha nemorella (L.) 55: light-trap 3 exx. 11.-18.8.1999.

Acrolepiopsis assectella (Zell.) 84-GO: 2 exx. 29.5.1992, 4.6.1996.

\section{Oecophoridae}

Semioscopis steinkellneriana (Den. \& Schiff.) 84-GO: 1 ex. 3.6.1996.

Depressaria sordidatella Tgstr. (=weirella Stt.) 61: 1 ex. 19.8.1997; 84-GO: 1 ex. no date.

Exaeretia ciniflonella (Zell.) 61: 3 exx. 11.9.1995, 7.6.1998, 4.9.1998; 74 : 1 ex. 22.-23.6. 1999.

Borkhausenia fuscescens (Hw.) 43-9: 1 ex. 22. 7.1994, 1 ex. 27.7.1999; 61: 4 exx. 25.6.1996, 19.7.1995, 10.8.1994, 18.7.1997.

\section{Elachistidae}

Biselachista kebneella (Traugott-Olsen \& Nielsen) 43-20: 1 ex. 24.7.1996. This species recently (1977) described from Swedish and Finnish Lapland is - to our knowledge (Falkovitsh 1981) - reported here for the first time from Russia. The supposed food plant is Luzula sp. (Traugott-Olsen \& Nielsen 1977).

\section{Coleophoridae}

Coleophora milvipennis Zell. 43-36: 1 ex. 26. 7.1995; 80: 1 ex. 26.7.1996. 
Coleophora vestianella (L.) 60: 1 ex. 3.8.1992; 61: light-trap, 1 ex. 20.8.1996.

\section{Gelechiidae}

Chrysoesthia sexguttella (Thnbg.) 60, 61, 83DE, 83-TS, 84-GO, 85-VE: common, locally abundant.

Teleiodes notatella (Hb.) 68: 1 ex. 21.6.1999.

Exoteleia dodecella (L.) 61: light trap, 1 ex. 9.8.1996

Chionodes electella (Zell.) 61: 1 ex. 19.7.1995.

\section{Tortricidae}

Pandemis cinnamomeana (Tr.) 60: 1 ex. 23.6. 1995.

Exapate congelatella (Cl.) 61: 1 ex. 26.9. 1998.

Acleris laterana (F.) 55: 1 ex. 18.8.1999.

Acleris notana (Don.) 43-29: 1 ex. 2.7.1998.

Acleris emargana (F.) 55: light-trap, 3 exx. 18.-23.8.1999; 61: light trap, 11 exx. 23.8-6.9. 1996-98.

Cochylis nana (Hw.) 44a: 600 m. 1 ex. 16.7. 1994.

Celypha rufana (Sc.) 83-A1, 83-A2, 83-AN, 83-GO, 83-SK, 83-TS, 85-VE: fairly common.

Apotomis fraterculana Krog. 43-9: 1 ex. 14.7.1997. This species is described from Lapland in 1945 by Krogerus. Russia (Kuznetsov, 1978 ) is not included in its distribution, but actually two syntypes are collected in territory nowadays belonging to Russia. One of the syntypes is collected from Lps Salmijärvi (locality no 9) in Kola Pns, but was overlooked by us in 1994, because the specimen is in a kept private collection.

Ancylis apicella (Den. \& Schiff.) 80: 1 ex. 26. 7.1996.

Epinotia solandriana (L.) 61: light trap, fairly common.

Epinotia brunnichana (L.) 61: light trap, 2 exx. 31.8.1996, 26.8.1997.

Epinotia maculana (F.) 61: light trap, 9 exx. 27.8-11.9.1995-97.

Epinotia demarniana (F. v. R.) 63: 1 ex. 24.6. 1995.

Epinotia nisella (Cl.) 39: 1 ex. 23.7.1998.

Eucosma hohenwartiana (Den. \& Schiff.) 61:
2 exx. 3.7.1994, 23.7.1998; 83-DE: 1 ex. 13.7. 1997.

Eucosma guentheri (Tgstr.) 68: 1 ex. 21.6. 1999; 72: 1 ex. 22.6.1999.

Blastesthia turionella (L.) 38-16: 1 ex. 20. 7.1994; 83-RJ: 1 ex. 6.7.1998.

Cydia indivisa (Danil.) 43-9: 1 ex. 12.7.1998.

\section{Pterophoridae}

Stenoptilia veronicae Karv. 61, 83-A2, 83-BS, 83-DE, 83-GO, 85-VE: common.

\section{Pyralidae}

Dioryctria schuetzeella (Fuchs) 80: 1 ex. 26.7. 1996.

Phycitodes binaevellus (Hb.) 61: 1 ex. 27.7. 1992. ic.

Ephestia kuehniella (Zeller) 60: synanthrop-

Ephestia elutella (Hbn.) 60: synanthropic.

Nymphula stagnata (Don.) 82-BV: 1 ex. 31 . 7.1998

Agriphila selasella (Hbn.) 61: 1 ex. 23.7.1998.

Eurrhypara hortulata (L.) 84-GO: 1 ex. 3.7. 1991.

Nomophila noctuella (Den. \& Schiff.) 83-AN: 1 ex. 29.7.1998.

\section{Pieridae}

Aporia crataegi (L.) 84-GO: 3 exx. 1990.

\section{Nymphalidae}

Polygonia c-album (L.) 84-GO: 1 ex. 12.9. 1992.

\section{Thyatiridae}

Thyatira batis (L.) 84-GO: 1 ex. 16.6.1991.

\section{Geometridae}

Scotopteryx chenopodiata (L.) 60, 84-GO, 85VE: common.

Coenocalpe lapidata (Hbn.) 61: $1 \mathrm{ex}$. 20.9.1998. 
Horisme tersata (Den. \& Schiff.) 84-GO: 1 ex. 27.6.1991.

Operophterabrumata (L.) 60, 61, 83-LO: common. Reared on Vaccinium uliginosum.

Operopthera fagata (Scharfenb.) 83-DE: 1 ex. 23.9.1989.

Plagodis pulveraria (L.) 61, 82-BV, 83-AN, 83-BL, 83-ML, 83-MM, 83-RJ: common.

Epione repandaria (Hufn.) 61: 3 exx. 24.27.8.1994; 84-GO: 1 ex. 31.8.1991.

Lycia hirtaria (Cl.) 84-GO: 1 ex. 13.6.1994.

Arichanna melanaria (L.) 61, 81-OL, 83-RJ, 84-GO: 7 exx.

\section{Lasiocampidae}

Lasiocampa quercus (L.) 66: 1 ex. 24.6.1995; 83-RJ: 1 ex. VII.1953; 84-GO: 1 ex. 1990.

\section{Notodontidae}

Pheosia tremula (Cl.) 84-GO: ex larva on Populus tremula 5.8.1993.

Pterostoma palpinum (Cl.) 61: 2 exx. 28.6. 1992, 13.6.1994.

\section{Lymantriidae}

Orgyia antiqua (L.) 84-GO: 1 ex. 24.8.1991.

\section{Noctuidae}

Catocala adultera Mén. 55: light-trap, 1 ex. 18.8.1999.

Callistege mi (Cl.) 84-GO: 1 ex. 1990.

Agrochola helvola (L.) 61: light trap, 3 exx. 5-7.9.1997.

Xanthia togata (Esp.) 61: light trap, common; 84-GO: 1ex VIII.1990.

Brachylomia viminalis (F.) 61: light trap, 11 exx. 27.8-6.9.1994-97.

Apamea furva (Den. \& Schiff.) 61: light trap, 1 ex. 1.-2.8.1998.

Celaena haworthi (Curt.) 61: 2 exx. 26.8.1981, 5.9.1997.

Xestia brunneopicta (Matsum.) 87: 1 ex. 10.7. 1999. This Central- and East-Siberian species was surprisingly found in 1983 in Northern Finland, province Ks (Mikkola et al. 1989). This locality is about a hundred kms southwest of our place no 87. So far not recorded from Sweden or Norway. Amphipoea fucosa (Frr.) 61: light trap, 11 exx.

24-28.8.1994-97.

Hydraecia micacea (Esp.) 61: light trap, 4 exx. 25.8-10.9.1997, 1 ex. 13.9.1998.

Orthosia gothica (L.) 60: 1 ex. 13.5.1991; 61:

2 exx. 4-14.6.1992; 84-GO: 1 ex. 29.5.1991.

Graphiphora augur (F.) 60: 3 exx. 16.6.1996, 15.8.1997.

Diarsia rubi (View.) 61: 2 exx. 16.7.1992, 7.7.1997.

Lycophotia porphyrea (Den. \& Schiff.) 43-41: 1 ex. 22.7.1996.

Chersotis cuprea (Den. \& Schiff.) 84-GO: 1 ex. 1991.

Cerastis rubricosa (Den. \& Schiff.) 84-GO: 1 ex. 1991.

\section{Confirmed occurence}

From the species listed below our information in the 1994 list was based on the literature only. Recent findings confirm the occurrence of these species in the region.

Nematopogon magnus (Z.) 63: 1 ex. 24.6. 1995.

Taleporia borealis (Wck.) 44a: 700 m, 1 ex., 17.7.1994.

Agonopterix heracliana (L.) 60, 61, 85: common.

Cydia coniferana (Saxesen) 83-RJ: 1 ex. ex larva 12.6.1992.

Leptidea sinapis (L.) 61: 2 exx. 30.6-3.7.1991; 84-GO: 2 exx. 1.-9.7.1992.

Polyommatus eumedon (Esp.) 61, 83-BL, 84GO: common but not numerous.

Vanessa atalanta (L.) 60, 61, 83-TY, 84-GO, 84-ST: occasional migrant.

Eupithecia indigata (Hbn.) 83-RJ, 82-BV.

Semiothisa liturata (Cl.) 83-RJ, 84-GO.

Semiothisa clathrata (L.) 84-GO, 85-VE: common.

Acherontia atropos (L.) 60: 2 exx VII.1954; 83-VR: 1 ex 27.6.1990.

Ptilodon capucina (L.) 84-GO: 1 ex VI.1990. Scoliopteryx libatrix (L.) 84-GO: 1 ex 1988. Autographa gamma (L.) 60, 61, 84-GO: oc- 
casional migrant.

Hada plebeja (L.) (=nana Hfn.) 61, 84-GO.

\section{Discussion}

Nearly all of the newly discovered species are common and widespread in Northern Fennoscandia and their occurence on the Kola Peninsula was more or less predictable. Biselachista kebneella (Traugott-Olsen \& Nielsen 1977) and Apotomis fraterculana Krogerus, 1945 are boreal species, described in the 20th century from Lapland, so they were expected as well. Tinea svenssoni Opheim, 1965 is described from Norway and Sweden and is fairly common in Finland. These three above mentioned species are reported here for the first time officially from Russia.

The lepidopteran fauna of the Kola Peninsula now numbers 676 species. Koponen \& Linnaluoto (1980) give a total of 602 species of Lepidoptera for northernmost Europe. The higher species number from Kola is due to the southern situation of the southernmost parts of the peninsula.

Acknowledgements. M. Kozlov thanks all the participants of the expeditions to Kola under harsh circumstances: Artem Blashkevitsh, Evgeni Melnikov, Elena Zvereva and Vitaly Zverev. E. V. Shutova thanks F. N. Shkljarevitsh for collecting in place no. 85 . We all want to express our gratitude to L. Kaila, J. Kullberg, M. I. Falkovitsh and V. I. Piskunov for identifying difficult taxa. Expeditions to Kola were funded by the Academy of Finland.

\section{References}

Falkovitsh, M. I. 1981: Elachistidae in Medvedev, G. S. (ed.) Identification keys to the Insects of the European part of the USSR vol. 4, part 2. (In Russian). - Leningrad 787 pp.

Jalava, J. 1980: Tineidae (Lepidoptera) from nests of the Ural owl (Strix uralensis Pall.). - Notulae Entomologicae 60: 96-100.

Karsholt, O. \& Razowski, J. (eds.) 1996: The Lepidoptera of Europe (A Distributional Checklist). - Apollo Books, Stenstrup $380 \mathrm{pp}$.

Kozlov, M. V. \& Jalava, J. 1994: The Lepidoptera of Kola Peninsula, Northwestem Russia. - Entomol. Fennica 5: 65-85.

Krogerus, H. 1945: Die Finnischen Apotomis- Arten. (Lep. Tortricidae). - Notulae Entomologicae XXV: 137-152.

Kuznetsov, V. I. 1978: Tortricidae in Medvedev, G. S. (ed.): Identification keys to the Insects of the European part of the USSR vol. 4, part . (In Russian), - Leningrad 711 pp.

Linnaluoto, E. T. \& Koponen, S. 1980: Lepidoptera of Utsjoki, northernmost Finland. - Kevo notes 5, 68 pp.

Mikkola, K., Sinervirta, M. \& Vaalamo, K 1989: Xestia brunneopicta (Matsumura) new to Europe (Lepidoptera, Noctuidae). - Notulae Entomologicae 69: 3336.

Opheim, M. 1965: The Norwegian species of Niditinea Petersen, 1957 and Tinea Linnaeus, 1758 (Lep., Tineidae). - Norsk Entomol. Tídskr. 13,1: 52-60.

Petersen, G. 1973: Tineidae II. Ergebnisse der zoologischen Forschungen von Dr. Z. Kaszab in der Mongolei. - Reichenbachia 14: 89-94.

Ponomarenko, M. G. 1997: Tineidae in Kononenko, V. S. (ed.): Identification Keys to the Insects of Russian Far East. (In Russian), - Vladivostok 539 pp.

Svensson, I. 1953: Eine Neue Art der Gattung Tinea Zeller (Lepidoptera). - Opuscula Entomologica 18: 225-227.

Traugott-Olsen, E. \& Nielsen, E. S. 1977: The Elachistidae (Lepidoptera) of Fennoscandia and Denmark. Fauna Entomologica Scandinavica 6: 299 pp.

Varis, V. (ed.) 1995: Checklist of the Finnish Lepidoptera. Sahlbergia 2, 1: $80 \mathrm{pp}$.

Zaguljaev, A. K. 1960: Tineidae, part 3 subfam. Tineinae. Fauna SSSR, Insecta Lepidoptera IV.3. - Moskva, Leningrad 267 pp. 